\title{
MIGRACIÓN Y TRANSICIÓN: HIJOS DE INMIGRANTES DE ORIGEN LATINOAMERICANO EN SU TRANSICIÓN DE LA ESCUELA AL TRABAJO EN CHILE ${ }^{1}$
}

\section{Migration and transition: children of Latinamerican inmigrants in their transition from school to work in Chile}

Kerstin Hein ${ }^{2}$

Universidad de Santiago de Chile, Santiago, Chile.

Recibido: 31 de diciembre de 2010. Aprobado: 7 de septiembre de 2011.

\section{RESUMEN}

Se presentan los principales resultados de un estudio cuyo objetivo fue comprender la forma en que hijos de inmigrantes de origen latinoamericano en Chile viven, entienden y manejan su transición de la escuela al trabajo. Con este propósito se realizaron entrevistas biográficas narrativas y evaluaciones cualitativas de las redes sociales de 13 hijos de extranjeros de origen latinoamericano en Chile. Los jóvenes fueron contactados por primera vez durante su último año de educación secundaria, y entrevistados por segunda vez un año después de haber abandonado el sistema escolar. Entre los resultados que se discuten se encuentra el proceso de migración de los entrevistados, su integración cultural en el país y su percepción de la distancia cultural. También se aborda la integración social y experiencias de discriminación que afectan a los jóvenes en el contexto escolar. Finalmente se presenta la evolución de todos estos aspectos un año después de que los participantes han egresado de la enseñanza media.

PALABRAS CLAVE: Hijos de inmigrantes, integración social y cultural, transición.

$1 \quad$ Ponencia presentada en el Congreso Interdisciplinario de Ciencias, Tecnologías y Culturas. Diálogo entre las Disciplinas del Conocimiento. Mirando al Futuro de América Latina y el Caribe, del 29 de octubre a 1 de noviembre 2010, en Santiago de Chile. Fondecyt Nº11085001 (2008): "Estudio biográfico interpretativo acerca de la significación y manejo de la transición de la escuela al trabajo por parte de jóvenes con trasfondo migratorio de origen latinoamericano en Chile".

2 Licenciada en Psicología por la Universidad Diego Portales, Santiago de Chile. Doctora en Psicología Social y Sociología por la Ludwig-Maximilans-Universität en Munich, Alemania. Actualmente es investigador en el Instituto de Juventud Alemán y es profesora del Doctorado en Psicología de la Universidad de Santiago de Chile (USACH). Correo electrónico: kerstinhein@gmail.com 


\begin{abstract}
The following article presents the results of a study that explored how children of Latin American immigrants in Chile experience, perceive and manage their transition from school to work. The study was based on biographical narrative interviews and qualitative evaluations of social networks of 13 children of Latin American parents living in Chile. The participants were first contacted during their final year of secondary school, and they were interviewed again one year after completing school. The results discussed in this article include the migration process of the interviewees, their cultural integration and their perception of cultural distance. Results regarding the social integration and discrimination experiences that affect these young people within the school system are also addressed. Finally, the evolution of all of these issues one year after the participants completed secondary education is presented.
\end{abstract}

KEYWORDS: Children of immigrants, social and cultural integration, transition.

\title{
I. INTRODUCCIÓN
}

A partir de la década de 1990, Chile comenzó a recibir un creciente número de extranjeros provenientes de la región latinoamericana, lo que causó inquietud entre la población chilena, no solo debido a la magnitud del movimiento de inmigración, sino también debido al nuevo perfil de las personas que llegaban a nuestro país.

El Departamento de Extranjería y Migración (2010) estima la presencia de alrededor de 352.000 personas extranjeras en nuestro país, lo que corresponde aproximadamente al dos por ciento de la población total. Esto constituye un importante aumento con respecto a lo señalado por el censo de 2002, siendo que ya entonces se registraba el número de inmigrantes más elevado en el contexto chileno desde 1952 (Martínez 2003a, 2003b).

Por otra parte, se estima que un 73 por ciento del total de extranjeros residentes en Chile corresponde a personas de origen sudamericano (Departamento de Extranjería y Migración 2010). Este hecho contrasta con el perfil de los movimientos migratorios registrados anteriormente a lo largo de la historia de Chile, los cuales estaban constituidos fundamentalmente por personas de origen europeo (CEPAL/CELADE 2006; Martínez 2003a, 2003b; Stefoni 2003). 
El nuevo perfil del inmigrante que llega a Chile ha otorgado visibilidad y un nuevo significado a los flujos migratorios actuales (Doña y Levinson 2004): Mientras que la inmigración europea es considerada mayoritariamente como un aporte al desarrollo nacional (Doña y Levinson 2004; Stefoni 2003; Ojeda-Ebert 1984; Salazar y Pinto 1999), se estigmatiza fuertemente a los inmigrantes provenientes de países sudamericanos, considerándolos delincuentes, pobres, inferiores y subordinados (Stefoni 2003). Esto ha contribuido a la percepción de que los movimientos migratorios actuales constituyen un problema social que debe ser sometido a escrutinio científico.

Si nos detenemos a revisar la conformación del grupo de extranjeros residentes en Chile, veremos que la mayor parte proviene de países fronterizos, especialmente de Perú (37,1 por ciento). Le siguen en importancia la inmigración Argentina (17,2 por ciento), Bolivia (6,8 por ciento) y Ecuador (5,4 por ciento) (Departamento de Extranjería y Migración 2010). La distribución espacial de estos grupos resulta distintiva: la Región Metropolitana (RM) concentra a más del 64 por ciento del total de inmigrantes, siendo estos mayoritariamente peruanos (Departamento de Extranjería y Migración 2010). La Región de Tarapacá acoge principalmente a los residentes de origen boliviano, mientras que los inmigrantes argentinos tienden a distribuirse de manera homogénea a lo largo de nuestro país (Martínez 2003a, 2003b; IOM/OIM 2004).

$\mathrm{Al}$ analizar los principales motivos de migración de los distintos grupos mencionados, es posible señalar que la inmigración argentina tiene un carácter más bien familiar (Martínez 2003a, 2003b; IOM/OIM 2004), mientras que los flujos de origen peruano, ecuatoriano y boliviano responden principalmente a motivos laborales (Departamento de Extranjería y Migración 2010; Martínez 2003a, 2003b). En estos grupos también es posible observar un predominio de la población femenina debido a la importante proporción de mujeres inmigrantes que busca insertarse en el servicio doméstico local (CEPAL/CELADE 2006; Martínez 2003a, 2003b; Stefoni 2003; Godoy 2007).

En cuanto a la inmigración colombiana, se estima que corresponde al 3,7 por ciento del total de la población de residentes extranjeros en Chile, siendo el grupo de migrantes más numerosos tras los ecuatorianos. Si bien la mayoría se incorpora a nuestro país en calidad de inmigrante, se trata del grupo de personas que más solicita el reconocimiento de la condición de refugiado en nuestro país (Departamento de Extranjería y Migración 2010). 


\section{SEGUNDA GENERACIÓN DE INMIGRANTES EN CHILE}

El estudio que se presenta a continuación se enmarca dentro del contexto antes señalado y tuvo por objetivo comprender la experiencia de los hijos de inmigrantes de origen latinoamericano en Chile desde una perspectiva subjetiva. El énfasis puesto en el origen latinoamericano se explica a partir de la situación de estigmatización que afecta primordialmente a este grupo de inmigrantes, convirtiendo el fenómeno migratorio en un problema de relevancia social.

Por otra parte, el interés por conocer más de cerca la situación de los hijos de inmigrantes responde a varias razones. En primer lugar se trata de un escenario incipiente, poco estudiado, pero de creciente interés público en nuestro país. Segundo, se ha visto que los efectos de una migración no terminan en la generación de personas que abandonan su país de origen para instalarse en otro lugar, sino que también afecta la vida de sus hijos, y en algunos casos incluso la vida de los hijos de los hijos. En este sentido se habla de la existencia de migrantes de segunda y tercera generación.

En rigor, cuando se habla de una segunda generación se piensa en los hijos de inmigrantes que nacieron en el país de acogida, o bien llegaron junto con sus padres a edades tempranas (Ward, Bochner y Furnham 2001; Portes y Rumbaut 2006). Estudios han demostrado que la experiencia de migración a edades muy tempranas (hasta aproximadamente el inicio de la edad escolar) no resulta biográficamente significativa para los sujetos involucrados (Hein 2006) y produce muy bajo nivel de estrés, mientras que en edades posteriores tiende a convertirse en un evento biográfico crítico (Bendit 1997).

Si bien no queda claro si niños y jóvenes que emigran con posterioridad al inicio de la edad escolar constituyen parte de la primera o segunda generación de inmigrantes, en el marco de nuestro estudio hemos preferido considerarlos parte de la segunda generación, ya que su experiencia se distingue cualitativamente de la de sus padres en al menos dos sentidos. En primer lugar, no eligen emigrar por cuenta propia, sino que son sus padres quienes deciden por ellos. En segundo lugar, su primera inserción en el país no ocurre a través del mercado laboral, sino a través del establecimiento escolar.

Si se considera la configuración de la población de origen extranjero de acuerdo al censo del año 2002 y con datos más recientes provistos por parte del Departamento de Extranjería y Migraciones (2010), se podrá apreciar que aún existe una proporción relativamente baja de niños y jóvenes inmigrantes sobre todo en aquellos grupos cuyo 
principal motivo de migración es de índole laboral. ${ }^{3}$ No obstante, se trata de un escenario de creciente importancia, sobre todo para aquellos establecimientos educacionales que reciben a los hijos de extranjeros en sus aulas.

\section{TRANSICIÓN DE LA ESCUELA AL TRABAJO}

Dentro del marco de nuestro estudio sobre hijos de inmigrantes de origen latinoamericano en Chile quisimos conocer específicamente cómo ellos viven, entienden y manejan la transición de la escuela al trabajo en Chile.

Por lo general, los estudios sobre la segunda generación de inmigrantes han tendido a focalizarse en la formación de identidades culturales en contextos multinacionales (Auernheimer 1988, 2007; Ward, Bochner y Furnham 2001; Teo 1994; Heckmann 1981), pero menos en el proceso de transición hacia la vida adulta (Bolzman, Fibbi y Vial 2005). No obstante, resulta relevante investigar la transición hacia la vida adulta, y en particular el tránsito desde la escuela al trabajo por parte de jóvenes inmigrantes por varias razones: una de ellas tiene relación con las dificultades en el rendimiento escolar que se ha visto presentan niños y jóvenes de origen extranjero a nivel internacional (Marbach 2006; Bendit 1997, 2006; Bechman y Moerch 2006; PISA 2006; Walther y Stauber 2002). También resulta relevante estudiar el tránsito de la escuela al trabajo, debido a que constituye un período especialmente sensible a la inclusión o exclusión de jóvenes en general, y de jóvenes con antecedentes de migración en particular (Walther y Stauber 2002; Bendit 2006).

El tránsito de la escuela al trabajo se encuentra estructurado por un conjunto de instituciones y patrones culturales que acompaña a los jóvenes en su camino hacia el mercado laboral. Dentro de este marco encontraremos distintos tipos de trayectorias: trayectorias académicas o de educación superior, trayectorias de educación técnica, trayectorias que atraviesan otro tipo de formaciones educacionales y trayectorias que se caracterizan por un ingreso directo de la escuela al trabajo sin obtener calificaciones adicionales (Walther y Stauber 2002).

En el caso de Chile, la Quinta Encuesta de Juventud (Instituto Nacional de la Juventud 2006) sugiere que la trayectoria más frecuente para jóvenes chilenos es el ingreso directo al mercado laboral tras finalizar la enseñanza media, ya que a partir de entonces

3 Se estima la presencia de un 15,34 por ciento de menores de 15 años entre la población extranjera en Chile, siendo peruanos, bolivianos y colombianos, quienes presentan el menor porcentaje de personas en este tramo etario (Departamento de Extranjería y Migraciones 2010). 
el trabajo comienza a desplazar los estudios como actividad principal. Sin embargo, se desconoce qué sucede con los hijos de inmigrantes una vez que terminan la educación secundaria en Chile.

En toda trayectoria es posible distinguir dos umbrales: el primero corresponde al paso desde la educación secundaria hacia una formación superior, ya sea técnica o universitaria. El segundo se refiere al término de la formación profesional o universitaria y el ingreso al mercado laboral propiamente tal (Instituto Nacional de la Juventud 2006). Durante los últimos años se puede apreciar la tendencia a centrar la atención en el primer umbral del proceso de transición, sobre todo debido a la creciente importancia que tiene la formación profesional o universitaria para incorporación al mundo del trabajo, y la creciente precariedad que significa una trayectoria directa al mercado laboral (Müller y Braun 2007; Reißig, Gaupp y Lex 2008).

Dentro del marco de nuestro estudio realizamos un seguimiento de la trayectoria de un grupo jóvenes de origen extranjero hasta un año después de haber abandonado la enseñanza escolar en Chile. Esto significa que fuimos capaces de conocer cómo ellos viven, entienden y manejan principalmente el primer tramo de su transición de la escuela al trabajo.

\section{METODOLOGÍA}

Se entrevistaron 13 jóvenes extranjeros en dos instancias. El primer contacto fue realizado durante cuarto medio. El segundo contacto fue realizado un año después con el fin de ver lo que sucede una vez que los jóvenes abandonan el sistema escolar. El principal canal de contacto con los jóvenes fueron los establecimientos educacionales ubicados en las comunas de Santiago, en donde se registra la mayor concentración de la población inmigrante, es decir Santiago, Recoleta, Independencia y Estación Central (Martínez 2003a; Stefoni et al. 2009). También se contó con el apoyo de la Oficina de Protección de la Derechos de la Infancia (OPD) de Recoleta y del Programa de Refugio de la Vicaría de la Pastoral Social.

El grupo de participantes estuvo compuesto por ocho peruanos, dos ecuatorianos y tres colombianos. Seis fueron mujeres y siete hombres. Sus edades fluctuaron entre los 16 y 19 años la primera vez que los contactamos. El tiempo de estadía en Chile osciló entre uno a siete años. En cuanto a la situación legal de los entrevistados, durante el primer contacto observamos cinco casos con residencia definitiva, seis con residencia temporal sujeta a contrato, y dos en condición de refugiados. Durante la fase de seguimiento, prác- 
ticamente todos habían obtenido la residencia definitiva, o bien se encontraban en el proceso de obtenerla. En todos los casos se entregaron y firmaron cartas de consentimiento informado, tanto para padres como para hijos.

De acuerdo con el propósito de conocer la experiencia de los participantes desde su propio punto de vista, se optó por realizar un estudio biográfico interpretativo, el cual permite reconstruir la historia de un individuo a partir del sentido que otorga el sujeto a su propia biografía (Cornejo 2006; Cornejo, Mendoza y Rojas 2008; Rosenthal 2005; Bertaux 2005).

Los datos fueron recolectados a través de entrevistas biográficas narrativas y evaluaciones cualitativas de redes sociales. La entrevista narrativa se caracteriza por ser una entrevista abierta, en la cual no se utiliza una pauta de temas para guiar la conversación, sino que se permite que sea el entrevistado mismo quien organice el encuentro de acuerdo con sus propios criterios de relevancia subjetiva (Rosenthal 2005; Rosenthal et al. 2006; Flick 2004; Hopf 2000; Lamnek 1998). La evaluación de redes sociales se realizó a través del método de evaluación EGONET-QF, el cual permite hacer visible la red social de un sujeto ubicando a las personas más significativas de la red social en círculos concéntricos más o menos alejados de un núcleo que representa al entrevistado (Straus 1994, 2002).

A partir de los datos recolectados se realizaron reconstrucciones de casos y análisis de contenidos. El primer procedimiento consiste en reconstruir la historia de vida de una persona en orden cronológico a partir de un análisis secuencial de los datos biográficos, un análisis secuencial del relato, y a partir de la comparación y posterior integración de la historia vivida (Rosenthal 2005; Rosenthal et al. 2006; Rosenthal y Fischer-Rosenthal 2000). El análisis de contenidos (Mayring 2003; Lamnek 1998; Rodríguez-Gómez, Gil y García 1996) fue realizado con el fin de facilitar la comparación de los casos, utilizando categorías desarrolladas a partir de la reconstrucción de cada biografía y en relación a los objetivos de investigación.

\section{RESULTADOS}

A continuación presentaremos algunos de los principales resultados obtenidos a lo largo de la investigación. Entre estos se encuentra, en primer lugar, el proceso de migración e integración cultural expresada a través de la percepción de distancia cultural, ambos aspectos estrechamente vinculados a la condición y experiencia de ser inmigran- 
tes. El proceso de migración e integración cultural constituyeron parte de las temáticas centrales de las primeras entrevistas realizadas con los participantes del estudio.

Luego abordaremos otro tópico central durante los primeros encuentros con los jóvenes inmigrantes, referido a su integración en el contexto escolar chileno. Aquí daremos especial importancia a las relaciones que se establecen entre chilenos y extranjeros, y a las experiencias de discriminación que se dan en el contexto escolar, lo que se encuentra en concordancia con los criterios de relevancia subjetiva establecidos por los mismos entrevistados.

Finalmente, damos a conocer algunos resultados de nuestras entrevistas de seguimiento, las cuales muestran la evolución que tuvieron nuestros participantes un año después de haber sido entrevistados por primera vez. Al respecto consideraremos tanto el desarrollo del proceso de integración cultural, como también los cambios que los jóvenes han experimentado con respecto a la discriminación de extranjeros. También presentaremos las trayectorias que nuestros entrevistados han emprendido tras abandonar la enseñanza media en nuestro país.

\section{1. "El peor viaje de mi vida"}

Para la gran mayoría de los jóvenes entrevistados, la experiencia de migración se caracterizó por ser un proceso que se extendió en el tiempo y que comenzó con una larga separación familiar. La separación familiar se produjo debido a que uno o ambos padres emprendieron primero solos el viaje al extranjero, para posteriormente regresar a buscar a sus hijos. Por lo general fue la mujer quien decidió emigrar primero, lo que confirma la así llamada tendencia de feminización de la migración internacional (CEPAL/CELADE 2006; Martínez 2003a, 2003b; Stefoni 2003; Godoy 2007).

El principal motivo para emigrar correspondió a razones económicas, ya sea de subsistencia, o bien por una insatisfacción con respecto a la actividad laboral o la calidad de vida en el país de origen. Cuando hubo dificultades económicas, estas frecuentemente derivaron de una separación de pareja, la que dejó a la mujer en una situación precaria que no le permitió sostener a su familia, por lo que decidió emigrar.

Una vez en Chile, la mayoría de las madres trabajaron como asesoras del hogar, lo que confirma la tendencia de mujeres migrantes a ocupar este nicho en nuestro mercado laboral. Cabe señalar que aproximadamente para la mitad de ellas, realizar labores de empleada doméstica significó asumir una actividad menos calificada en comparación con las 
actividades realizadas en el país de origen, lo que da cuenta del proceso de descalificación que afecta a muchas mujeres durante el proceso de migración (Gutiérrez-Rodríguez 1999).

La separación familiar debido a la migración varió entre pocos meses hasta siete años, con un promedio de aproximadamente tres años de separación. Durante este tiempo casi todos nuestros entrevistados permanecieron con la abuela materna, lo que hizo que esta se convirtiese en una figura de cuidado muy importante en su vida. La abuela asumió el rol de madre sustituta aun cuando el padre de los menores se encontraba presente, lo que refleja la existencia de una concepción tradicional de los roles de género. "Aparte que es mi única abuela y es en quien yo confío más y es la que yo más quiero, o sea, mi abuela es como mi mamá." (Javiera, 16 años, peruana)

Con frecuencia, la abuela materna vivía muy cerca o incluso en la misma casa en la que vivían sus nietos, por lo que asumió funciones de cuidado mucho antes de la partida de la madre al extranjero. Esto contribuyó a que la migración de la madre no representase un quiebre sustancial en la vida de los entrevistados, lo que sí sucedía cuando los hijos debían separarse de sus abuelos.

Bueno, viví con mi abuela y después empezaron los problemas familiares. Cuando mi mamá apareció, me dijo que se iba a venir conmigo acá, que quería estar conmigo, y yo no podía aceptar eso porque para mí, mi mamá era mi abuela. (Javiera, 16 años, peruana)

Tal como mencionamos anteriormente, la segunda generación de inmigrantes normalmente llega al país de acogida como menores de edad en compañía de sus padres, siendo la migración familiar una decisión de los progenitores y no de los hijos. Esto implica que la migración adquiere un sentido de exilio para los hijos, sobre todo cuando ellos no son capaces de desarrollar una motivación propia con respecto al traslado de un país a otro. Esta situación se vio reflejada en la actitud que tuvieron nuestros entrevistados ante la migración a Chile, la cual varió entre la neutralidad y la aceptación pasiva hasta el rechazo extremo. Esto hizo que los padres se viesen obligados a desplegar todo tipo de mecanismos para seducir y convencer, y si eso no resultaba, obligar a sus hijos a reunirse con ellos.

Y yo no quería venirme, si de hecho tuve como depresión por la tristeza de separarme de las personas que quería demasiado [...] Es que mi mamá llegó y dijo: me los llevo, y me los llevo. Y mi abuela no pudo hacer nada. Yo de hecho no quería venirme, de hecho yo me escondía. Mi mamá atrasó el viaje como un mes: siempre me escondía, desaparecía, y ahí mi abuela dijo: "No, déjalo", y mi Mamá: "No, que no lo quiero dejar, 
porque después va a ser demasiado tarde para que se acostumbren a vivir conmigo".

(Claudio, 18 años, peruano)

Sí, y con mi papá y yo en mi caso, lloraba pero porque sabía que iba a extrañar, que algo malo iba a pasar. (Bruno, 17 años, ecuatoriano)

Tal actitud por parte de los hijos parece haber estado vinculada a dos aspectos. El primero tiene que ver con la larga separación y desvinculación afectiva entre padres e hijos. Con frecuencia los hijos desconocieron a los padres como apoderados y atribuyeron ese rol a su abuela. Entonces, ir a vivir con los padres al extranjero no era solamente adaptarse a un país distinto, sino también aprender a vivir en un contexto familiar con padres que en la práctica eran desconocidos. Incluso, en algunos casos la separación familiar fue percibida como franco abandono. Cuando esto fue así, se estableció una relación extremadamente conflictiva entre padres e hijos.

El segundo aspecto que condicionó la actitud negativa ante la migración a Chile tuvo relación con la resistencia a abandonar el mundo conocido hasta entonces. Aquí hay que considerar que la edad de los entrevistados al emigrar varió entre los 12 a 16 años. Esto significa que la gran mayoría de los participantes se encontraba en plena adolescencia, considerada como el momento más sensible y crítico en la vida de un individuo para emigrar de un país a otro (Bendit 1997).

Por lo general, el viaje a Chile fue realizado por tierra y descrito como agotador. En algunos casos se recordaron detalles muy vívidos del viaje y la llegada a Chile, lo que hace pensar que el viaje mismo fue un evento que despertó fuertes emociones.

\section{Experiencia de distancia cultural}

Una de las experiencias subjetivas más fundamentales vinculadas a una migración es la experiencia de distancia o diferencia cultural en el país de acogida. En el marco de nuestro estudio entendimos la experiencia de distancia cultural como una interpretación subjetiva del individuo. Esto quiere decir que no se trata de una diferencia cultural objetiva medida a través de la distancia que existe entre una cultura nacional y otra (Ward, Bochner y Furnham 2001), sino de una interpretación subjetiva e individual basada en la percepción de distancia cultural y la experiencia de extrañeza que surge en el encuentro con elementos culturales que contrastan con los referentes culturales que habían resultado familiares hasta entonces (Suanet y Van de Vijver 2009; Hein 2006). 
Al respecto, Suanet y Van de Vijver (2009) han demostrado que es la percepción subjetiva de distancia cultural, y no la distancia cultural objetiva, la que determina la angustia que viven los inmigrantes durante su proceso de aculturación en el país de acogida. Personas que perciben una baja distancia cultural entre su cultura de origen y la cultura del país receptor, tenderán a presentar un mejor ajuste psicológico, menor grado de nostalgia y una mayor disposición al contacto social con personas del país de acogida.

En los relatos analizados la experiencia de diferencia cultural se expresó fundamentalmente a través de la comparación de lugares y personas, mientras que no constituyó un tema relevante cuando se trataba de la comprensión y orientación según las normas y los valores de nuestro contexto, y mucho menos aún en términos del dominio de la lengua nativa de nuestro país. Esto último sin duda constituye un factor que favorece la integración de inmigrantes de origen latinoamericano en Chile.

Al comparar el aquí con el allá se tendió a considerar que Chile era un país más moderno y avanzado en comparación con los respectivos países de origen. Por otra parte, Chile era retratado como un lugar en el cual se puede obtener bienestar material, pero no emocional, lo que se vio reflejado en el contraste que realizaron los participantes entre Chile como un lugar frío y los países de origen como lugares cálidos. Si bien esta comparación muchas veces comenzó haciendo alusión a las condiciones climáticas de los lugares, muy pronto pasó a expresar las características del ambiente social que los jóvenes percibían en nuestro país.

¿Qué no me gusta de Chile? No me gusta el frío, no me gusta el invierno, me aburro demasiado, me da desesperación porque en la parte de Colombia donde yo vivo hace bastante calor, son unos 38 grados todo el año, entonces cuando llego acá hace demasiado frío. Estoy pero no me dan ganas ni de salir, ni levantarme de mi cama. Cuando llega el verano me encanta [...] No sé, igual la gente allá es más así como que si pone música, pone música para todos. Entonces hay mucha música, hay mucha fiesta, los fines de semana son muy prendidos y acá, o sea la diferencia con Chile es el frío que no lo soporto. Yo creo que igual yo estoy acostumbrada a lo caliente, pero no sé el frío me aburre. (Natalie, 17 años, colombiana)

Ahora bien, el contraste entre el contexto chileno como inhóspito, exigente y racional, y los países de origen como lugares alegres, festivos y espontáneos fue mencionado con mayor frecuencia durante el primer contacto con los entrevistados, mientras que prácticamente desaparece durante el seguimiento de los casos. Como veremos a conti- 
nuación, esto parece encontrarse en estrecha vinculación con las experiencias de discriminación en el contexto chileno.

Para la mayoría de los entrevistados, la comparación entre personas chilenas y nativos del país de origen ocupó un lugar destacado en los relatos. Al respecto cabe recordar que el primer lugar de encuentro de los participantes con la cultura chilena ocurre en el contexto escolar, por lo que no sorprende que la experiencia de diferencia cultural gire en torno a la diferencia que perciben entre ellos y sus compañeros chilenos.

Existe una palabra que resume la visión que los entrevistados tuvieron de la juventud chilena: decadencia. A ojos de los participantes, sus compañeros chilenos son decadentes porque son rebeldes, contestatarios y sobre todo poco respetuosos hacia la autoridad, lo que sorprendió y molestó a los jóvenes de origen extranjero. Esta apreciación lleva a pensar que la relación entre adultos y jóvenes se estructura de manera más conservadora en los respectivos países de origen, y que los adultos son vistos como figuras a las cuales uno debe respeto y obediencia.

Con los profesores... ya que aquí no está bien... ehh... como le dicen acá... bien... eh, no le enseñan muy bien a los jóvenes aquí a respetar a los profesores [...] Irrespetuosos en todo el sentido de la palabra. (Cristóbal, 16 años, colombiano)

Otra cosa es que los alumnos son malcriados y no le tienen respeto al profesor. (Jorge, 16 años, peruano)

Lo decadente de la juventud chilena también fue vinculado a su forma de ser más liberal. Este último punto aludía especialmente al caso de mujeres chilenas que usan el uniforme demasiado corto, se maquillan en exceso o se visten con prendas llamativas. Este comportamiento fue comparado con la manera de ser más recatada, discreta y tímida de las jóvenes en los países latinoamericanos de origen, y calificado como "poco lady".

Como que acá en Chile es como más abierto al público, allá no [...] Y como que eso no es apropiado en mi país, el maquillaje, como que las niñas son así. [...] el uniforme, abajo de la rodilla y acá no [...] Pero como que la mujer debe ser así, como sin pintura como que no puede estar mostrando todo y acá no po, como que son más liberales. (Javiera, 16 años, peruana)

Los hombres chilenos, por su parte, fueron percibidos como groseros, poco educados y poco esforzados. "No sé si serán todos los colegios... yo creo que no... pero en 
este colegio en especial los alumnos la gran mayoría son muy perezosos... flojos" (Cristóbal, 16 años, colombiano).

Porque como que hablaban muchos garabatos aquí, como que hablan mucho, mucho a la madre y como que eso en realidad se me pegó un poco, pero yo trato igual un poco de no; es que hablan mucho garabato aquí los jóvenes chilenos, no respetan a los profesores, son un poco rotos para comer, porque en la comida se... la madre, se tiran panes, se tiran comida, eh, se tiran eructos en la mesa. (Jorge, 16 años, peruano)

En general cabe señalar que existía poca comprensión por la cultura juvenil, las formas de diversión y expresión juvenil en Chile, especialmente cuando se trata de jóvenes que pertenecen a tribus urbanas.

Para empezar en Colombia, los hombres son los hombres y no tienen por qué andar con aritos en las orejas. No tiene que andar con el pelo largo y todo ese cuento, y el cuento de los pantalones abajo yo veía eso como no sé, o sea yo no tengo que andar viendo cómo se viste otra persona pero lo de los pantalones abajo no sé son como cosas raras [...] Las tribus son muy raras, y otra cosa: el cuento de las lesbianas. (Natalie, 17 años, colombiana)

Resulta importante señalar que la mala opinión que los entrevistados manifestaron en relación a sus pares chilenos estuvo prácticamente restringida al ámbito escolar y que desaparece casi por completo una vez que los jóvenes abandonaron el liceo. Esto nos lleva a pensar que la negativa percepción de los compañeros se encuentra en estrecha vinculación con el maltrato y la discriminación que los participantes experimentan en el contexto escolar. Volveremos sobre este punto un poco más adelante.

\section{Integración en el contexto escolar chileno}

Tal como lo hemos manifestado anteriormente, el contexto escolar chileno representa el primer lugar en el cual los entrevistados se encuentran con chilenos y la cultura chilena. Por esta razón, resulta particularmente preocupante el hecho de que la experiencia escolar en Chile estuviese atravesada por diversas situaciones de maltrato y discriminación, ocupando un lugar destacado en los relatos que los entrevistados realizaron con respecto a su experiencia escolar. 
En cuanto al rendimiento escolar de nuestros entrevistados, es posible señalar que este osciló entre un rendimiento promedio y un rendimiento de excelencia, lo que contrasta con hallazgos vinculados a personas con trasfondo de migración a nivel internacional, mientras que resulta consistente con lo que se ha visto en relación a la inserción de niños y niñas migrantes en Chile (Stefoni et al. 2009).

Uno de los momentos más importantes en la experiencia escolar lo constituía el primer día de clases. Al respecto llamó la atención que casi todos lo relataron de la misma manera: los jóvenes comenzaron el día desorientados debido a diversos factores, como la inseguridad con respecto a la forma de vestirse, la hora de llegada o la sala a la cual debían dirigirse. Una vez que superaron esta desorientación inicial y los recién llegados encontraron sus respectivas salas de clases, los entrevistados contaron acerca de su presentación ante el curso. Esta resulta importante, pues da cuenta del tipo de acogida inicial que recibieron los participantes en el colegio. Posteriormente ocurría algo llamativo, y que es que los jóvenes debían sentarse al lado de un compañero poco popular o marginal al interior del curso. Con frecuencia se trataba de otro alumno extranjero, aunque también hubo relatos acerca de compañeros alocados, feos o gordos. La clave estaba en que este compañero era el blanco preferido de burlas y malos tratos por parte del curso.

Claro, entonces me senté al costado de un compañero gordito. Es feo decirlo, pero todos mis compañeros dicen que es feíto, pero yo no sé, yo encuentro bien agradable y me senté a su costado así para hacer amistad... y lo molestaban mucho, le tiraban papelitos a él, le tiraban naranjas podridas, le tiraban; entonces cuando yo veía eso que pasaba, me cambié de lugar. Igual no me llegaban a mí las cosas, porque a mí me respetaban, no me decían nada. (Jorge, 16 años, peruano)

Resulta interesante observar que los entrevistados manifestaron de manera sistemática, que eran sus compañeros de banco, quienes eran el blanco de los comportamientos de discriminación, mientras que ellos - por motivos inciertos- se salvaban de la situación. Esto resulta curioso, pues los entrevistados frecuentemente compartían la condición de extranjero, además que se sentaban al lado del "otro discriminado".

Al principio en el colegio me querían pegar como a la niña peruana, pero a mí nunca me han discriminado. Hubo una niña que la llegaron a golpear porque era buena; yo creo que eso influyó mucho también, gracias a Dios nunca pasó nada. (Natalie, 17 años, colombiana) 
La discriminación del otro constituyó la principal forma a través de la cual los entrevistados dieron cuenta acerca de las experiencias de discriminación en el contexto escolar. Es decir, la mayoría de las veces señalaron que eran otros quienes sufrían de maltrato, mientras que a ellos no les sucedía nada.

Es posible interpretar esta situación de distintas maneras. Por un lado es posible pensar que los entrevistados efectivamente eran víctimas de una forma moderada de maltrato, aunque creemos que es poco probable debido a varias razones: las situaciones de discriminación de los demás eran bastante intensas, lo que lleva a la siguiente pregunta: ¿qué es lo que determinaba la diferencia entre los participantes y los "otros discriminados"? Por otra parte, los entrevistados mostraron una elevada preocupación por el maltrato, lo que hace pensar que ellos sí fueron víctimas de la discriminación, pero no lo admitían para evitar verse a sí mismos como víctimas. Dicho de otra manera, el recurso narrativo del "otro discriminado" constituía una estrategia de afrontamiento ante la discriminación.

Como consecuencia de ser testigos de la discriminación del "otro", los entrevistados comenzaron a preocuparse por el tema de su visibilidad como extranjeros ante los demás. La mayoría atribuyó su visibilidad a su forma de hablar. En este sentido pensaron que eran reconocidos por su acento, por usar palabras distintas o por el hecho de no comprender o no utilizar los modismos chilenos. "Es que igual, hay diferencias: uno porque es de otro país, habla distinto, tiene diferentes dialectos por la forma de hablar, y por sus propias costumbres" (Julio, 17 años, peruano).

En su versión más moderada, la discriminación de extranjeros se expresó a través de molestias, burlas o imitaciones, las cuales tendían a ser constantes y sistemáticas, causando irritación entre los entrevistados.

A mí no me molestan, pero a mí igual me tiraban tallas, me decían: "Oye, peruano, ¿cómo es este país así, como es la armada? Igual me dicen: "Oye, vamos a ir a guerra con tu país". Me dicen así, ja, ja... pero a veces habían tallas que se metían con la familia y no me gustaba, que se metieran con la familia. (Jorge, 16 años, peruano)

En otros casos, la discriminación se expresó a través del maltrato directo, el cual incluía insultos, amenazas de golpe, incitaciones a pelear, o bien lo contrario, es decir, no tomar en cuenta y aplicar la "ley del hielo".

Porque los cabros chicos son... molestosos, o de repente compañeros que me tocaban en primero, pero nunca los pesqué, y siempre cuando no los pescas te molestan más, típico picao a choro. No los pesqué, y en vez de ponerme a pelear no los pescaba y me iba: eso. (Claudio, 18 años, peruano) 
He tenido varias experiencias negativas, te humillan, te marginan. (Carlos, 17 años, peruano)

La primera y principal reacción ante la posibilidad de ser discriminado consistió en permanecer callado, tratar de no llamar la atención y aislarse de los compañeros. "Igual yo no hablaba mucho por el cuento que cuando uno habla la gente se quedaba mirando y como que todos se callan y eso me intimida un poco. Entonces yo prefería no hablar" (Natalie, 17 años, colombiana).

Con el tiempo, los entrevistados se mostraron capaces de desarrollar estrategias de afrontamiento más activas y efectivas. La más común fue asimilarse a la cultura juvenil chilena, lo que incluía aprender a hablar como chileno. "Yo no hablaba ni raro, ni como peruano ni como chileno, pero se me pegó demasiado rápido el acento de acá. Después de dos meses como que ya estaba hablando así" (Claudio, 18 años, peruano).

A pesar de todo, la mayoría de los entrevistados logró establecer amistades con compañeros chilenos, aunque estas relaciones nunca llegaron a ser tan cercanas ni tan significativas como las establecidas con los pares de origen extranjero. En este sentido, aun las mejores amistades con chilenos no tendían a ir más allá de la jornada escolar, es decir, permanecían restringidas al contexto escolar.

Probablemente sea posible explicar este fenómeno a través de la imagen negativa que los entrevistados tenían de la juventud chilena. Por otra parte, también hay que considerar que los participantes tendían a sentirse irritados por las constantes molestias y "malos tratos" recibidos por parte de sus compañeros chilenos, lo que incidió negativamente en la relación con ellos.

\section{Un año después: desaparece la percepción de discriminación}

A continuación veremos cómo evolucionaron los temas expuestos anteriormente una vez que los participantes del estudio abandonaron la enseñanza media. Es decir, revisaremos si aún existe la experiencia de distancia cultural y cómo ella se expresa. También analizamos qué sucedió con las experiencias de discriminación, si continuaron o no, y de qué forma lo hicieron. 
Mencionábamos anteriormente que la experiencia de diferencia cultural se expresaba especialmente a través de la comparación entre el aquí y el allá, y a través del contraste entre nosotros y los otros. Durante las entrevistas de seguimiento fue posible apreciar que este tipo de comparaciones desaparecieron prácticamente por completo en todos los relatos. En este sentido, se pudo observar que la comparación entre Chile y los países de origen dejó der ser un tema de preocupación.

Algo similar ocurrió con la percepción negativa que los entrevistados expresaron con respecto a sus compañeros de colegio durante la primera fase de la investigación. En este sentido, durante las entrevistas de seguimiento los jóvenes mostraron una percepción mucho más diferenciada de sus compañeros de estudios o sus colegas de trabajo. Cuando los entrevistados daban cuenta de la existencia de algún tipo de rechazo hacia sus compañeros de estudios o colegas de trabajo, este se encontraba dirigido hacia personas o grupos puntuales al interior del curso o del trabajo, pero no al curso entero o a los jóvenes chilenos en general.

También se pudo apreciar la existencia de opiniones muy positivas, según las cuales compañeros o colegas de trabajo eran descritos como personas respetuosas y colaboradoras. Los participantes tendieron a explicar esta diferencia de actitud entre sus compañeros de estudio y sus antiguos compañeros de colegio a partir de la mayor edad y madurez de los primeros en comparación con los segundos.

Pero es distinto el instituto del colegio, porque veo que en el instituto como que todos son más amigables, no son como en el colegio, que son todos como cabros chicos [...] Todas las personas en el instituto son maduras, son centradas, se apoyan. Ahí se ve la diferencia entre los jóvenes chilenos y los jóvenes chilenos que son como... como que no piensan. Y eso me sorprendió, porque recibí una gran alegría, recibí su cariño. Y eso me gustó, por eso estoy estudiando. Me gusta. Y no me he sentido discriminado. Me he sentido en familia. (Seguimiento: Jorge, 17 años, peruano)

En concordancia con lo anterior, fue posible observar la desaparición de la percepción de discriminación debido al origen extranjero. Al respecto es importante subrayar que se trata de la percepción subjetiva de tal experiencia y no necesariamente de la ausencia real de discriminación. No obstante, la percepción de la falta de discriminación fue suficiente para hacer desaparecer la irritación y la sensación de amenaza presentes durante el año anterior en todos los entrevistados. También incidió positivamente en el bienestar subjetivo de los jóvenes y en el deseo de permanecer en el país. 
Cuando hubo experiencias de discriminación, estas ocurrieron de preferencia en el contexto laboral en vez del contexto educacional, siendo situaciones más bien aisladas y moderadas. Esto quiere decir que ya no se detectaron maltratos directos como insultos o golpes, sino solamente bromas, que, si bien molestaron a los entrevistados, no implicaron la misma intensidad, ni la misma sensación de amenaza que provocaban las situaciones de discriminación en el contexto escolar. Por otra parte, se diversificaron las razones por las cuales los participantes dijeron sentirse discriminados, siendo la condición de extranjeros solo una de ellas. Dicho de otra manera, más que discriminación por ser extranjero se observaron situaciones de discriminación debido a la edad, al género o al nivel socioeconómico.

En conjunto con la falta de percepción de discriminación también desapareció la intensidad y frecuencia con que los jóvenes enfatizaban el origen nacional. En cambio, apareció con más fuerza la identificación con el hecho de ser joven en Chile. En otras palabras, el ser joven y pasó a ser más importante que el ser extranjero.

Para nosotros ser mayor de edad es como empezar a tomar las cosas por tu propia mano y a empezar a individualizarse en tu vida, así que yo sé que tengo que buscar las cosas por mí misma. (Seguimiento: Natalie, 18 años, colombiana)

\section{Trabajar y estudiar}

En concordancia con el objetivo de comprender la forma en que los hijos de inmigrantes de origen latinoamericano en Chile significan y manejan su transición de la escuela al trabajo, analizamos finalmente los recorridos realizados por nuestros entrevistados durante el transcurso de un año tras abandonar la educación secundaria. Aunque no podemos decir con certeza qué tipo de trayectoria será la que los jóvenes entrevistados realizarán finalmente, sí podemos indicar el tipo de trayectoria que iniciaron tras terminar la enseñanza media.

Durante la formulación del proyecto, y de acuerdo con los datos arrojados por la Quinta Encuesta de Juventud, se definió el término de la educación secundaria como un punto de quiebre en la mayoría de las trayectorias biográficas de los jóvenes chilenos, debido que a partir de entonces el trabajo pasaba a desplazar el estudio como actividad principal (Instituto Nacional de la Juventud 2006). En efecto, fue posible observar que el término de la enseñanza media constituyó el momento a partir del cual el trabajo pasó a ser una actividad al menos tan importante para los jóvenes como el estudio. Sin embar- 
go, el trabajo no desplazó al estudio. Esto significa que los jóvenes no solo se dedicaron a estudiar, o no solo se dedicaron a trabajar. Al menos para la mayor parte de nuestra muestra, estudiar y trabajar fueron dos actividades que no pudieron ser consideradas la una separada de la otra.

Concretamente, observamos a tres jóvenes dedicados por completo a los estudios, a tres jóvenes dedicados solamente a trabajar, mientras que el resto realizaba ambas actividades. Dentro de este marco, trabajar constituía una necesidad, tanto como aporte a la economía familiar como también para financiar los estudios. Esto contrastó con el significado atribuido al trabajo el año anterior, en donde la actividad laboral fue asociada más bien al aprendizaje y desarrollo personal.

Ante la decisión de estudiar o trabajar, los jóvenes preferían trabajar. No solo porque el trabajo constituía una mayor fuente de satisfacción personal, sino también porque la posibilidad de estudiar se encontraba supeditada a la posibilidad de generar los ingresos necesarios para financiar los estudios. Además se pudo observar que tanto padres como hijos compartían la expectativa de que los jóvenes debían trabajar y aportar con sus ingresos a la economía del hogar una vez finalizada su educación escolar. De este modo, el hecho de trabajar y de percibir un sueldo incidía de manera positiva en las relaciones familiares, especialmente en el vínculo entre padres e hijos.

Los estudios superiores fueron considerados como una actividad menos atractiva y satisfactoria que el trabajo. Su valoración estuvo fuertemente vinculada al apoyo familiar con respecto al hecho de estudiar, lo que a su vez estuvo estrechamente relacionado con el nivel educacional alcanzado por los padres.

Tal como mencionamos anteriormente, durante las entrevistas de seguimiento la mayor parte de nuestra muestra se encontraba trabajando y estudiando al mismo tiempo, lo que constituye un dato relevante, pues las políticas de juventud tienden a considerar la educación como actividad principal de los jóvenes, mientras que no le otorgan la misma importancia a la inserción laboral (Hein y Cárdenas 2009). Cuando hacen esto tienen en mente la imagen del estudiante universitario que se dedica por completo a los estudios y dejan de lado el hecho de que muchos estudiantes deben trabajar para poder pagar su formación.

Siguiendo la misma lógica de razonamiento, las políticas de juventud también tienden a considerar educación y trabajo como instancias separadas, por lo que promueven la inserción en educación sin considerar la integración al mundo laboral, y viceversa. En cambio, de acuerdo a lo que nosotros pudimos apreciar a través de nuestra investigación, trabajo y estudio son dos actividades que no pueden comprenderse separadamente: 
los jóvenes necesitan estudiar para acceder a un mejor trabajo, a la vez que necesitan trabajar para poder pagar su formación, por lo que es necesario encontrar la forma de mejorar la compatibilización de ambas actividades.

Quienes trabajaron y estudiaron al mismo tiempo se sometieron a un horario de actividades bastante estricto durante la semana. La estrategia más común fue trabajar jornada completa durante el día y estudiar en horario vespertino durante la noche. La falta de tiempo libre durante la semana fue evidente, lo que incidió de manera notoria en la amplitud y calidad de las redes sociales de los afectados, así como en el nivel de cansancio debido a la sobrecarga de actividades.

\section{Trayectorias de formación técnica}

Como era de esperarse, fueron contadas las trayectorias que se podrían definir como académicas. De hecho, solo dos de los participantes lograron ingresar a la universidad, mientras que la mayoría de la muestra inició una trayectoria de formación técnica. También fue posible observar una trayectoria que podemos calificar de semicalificada. Se trata de un joven que se ha insertado en el mercado laboral gracias a su título de técnico medio. Consideramos que este tipo de trayectoria no alcanza a ser realmente una formación técnica propiamente tal, aunque representa una mejor preparación para el mercado laboral que un ingreso directo tras finalizar la educación media científico-humanista.

Finalmente, hubo dos casos que posiblemente se encuentren realizando un ingreso directo al mercado laboral, lo cual es considerado como la forma más precaria de insertarse al mundo del trabajo (Walther y Stauber 2002). Sin embargo, no es algo que sea posible afirmar con certeza, pues no sabemos cómo se desenvolverán estos casos en el futuro, si volverán a estudiar en algún momento, o si abandonarán por completo la idea de realizar alguna formación profesional.

\section{CONCLUSIONES}

Hemos realizado un recorrido por los principales resultados de un estudio cuyo principal objetivo fue conocer la manera en que los hijos de inmigrantes de origen latinoamericano viven, entienden y manejan la transición de la escuela al trabajo en Chile. Con este fin se realizaron entrevistas biográficas narrativas con 13 jóvenes extranjeros durante su último año 
de enseñanza media, y entrevistas de seguimiento un año más tarde con los mismos jóvenes con el propósito de conocer cómo les fue tras terminar la educación secundaria.

Entre los resultados destaca el relato que los entrevistados realizaron con respecto a su proceso de migración, el cual habitualmente comienza con una larga separación entre padres e hijos, y una posterior migración que es percibida por los participantes como un exilio. Los jóvenes lamentan especialmente el tener que separarse de los abuelos que los cuidaron durante el largo período de separación familiar.

Otro aspecto que se discutió fue la experiencia de distancia cultural, la cual fue definida como la interpretación subjetiva e individual de la distancia cultural y la experiencia de extrañeza que surge en el encuentro con elementos culturales que contrastan con los referentes culturales que habían resultado familiares hasta entonces. La percepción de distancia cultural se expresó principalmente en las narraciones a través de comparaciones entre lugares y personas.

La comparación entre lugares y personas realizada por los entrevistados retrató a Chile como un país moderno y avanzado, pero también inhóspito y frío. Asimismo, se observó una opinión muy negativa de los chilenos, con lo cual los entrevistados se refirieron casi exclusivamente a sus compañeros de colegio. Estos eran vistos como liberales, malcriados y en general poco respetuosos hacia la autoridad.

La mala opinión sobre la juventud chilena apareció estrechamente vinculada a la experiencia escolar de los participantes, atravesada por intensas y frecuentes situaciones de discriminación. En general, los entrevistados decían no ser víctimas, sino más bien testigos del maltrato de otros extranjeros, a quienes denominamos como los "otros discriminados". La forma en que se presentaron las situaciones de discriminación y maltrato en el contexto escolar hacen suponer que los entrevistados efectivamente eran objeto de sistemáticas burlas, amenazas de golpes y golpes por parte de sus compañeros de colegio, pero que no deseaban verse a sí mismos ni presentarse ante otros como víctimas de este tipo de situaciones. Es importante considerar este aspecto, pues podría llevar a una subvaloración y consecuente minimización de la gravedad de las situaciones de discriminación y maltrato que sufren los hijos de inmigrantes en los contextos escolares.

Las experiencias de discriminación afectaron las relaciones entre chilenos y extranjeros en el contexto escolar. Aunque la mayoría de los entrevistados logró establecer amistades con chilenos, estos vínculos nunca llegaron a ser tan cercanos ni significativos como las relaciones establecidas con otros extranjeros. Incluso las mejores amistades con 
chilenos no lograron trascender la jornada escolar, ni tampoco sobrevivieron al término de la educación media.

Curiosamente, un año después desapareció casi por completo la percepción de discriminación. Es decir, una vez que los jóvenes abandonaron el sistema escolar dejaron de percibir que eran discriminados por ser extranjeros. Este efecto fue independiente del tiempo de permanencia en Chile e independiente de la actividad que realizaban las personas. Es más, algunos entrevistados incluso manifestaron opiniones muy positivas sobre compañeros de estudio o colegas de trabajo, según sea el caso.

Si bien la falta de la percepción de discriminación no significa necesariamente que ya no exista la discriminación de extranjeros, sí incide de manera fundamental en el bienestar subjetivo de los individuos. La percepción de aceptación social, sea imaginada o real, fue determinante en la desaparición de la comparación que realizaban los entrevistados entre lugares y personas. Esto hace suponer que ya no existía una percepción de distancia cultural, o al menos que esta se había vuelto irrelevante para los jóvenes. De manera concordante también desapareció la necesidad de enfatizar el origen nacional, mientras que apareció el deseo de permanecer y radicarse en Chile.

Estos resultados nos deben hacer reflexionar acerca de las importantes y negativas consecuencias que tiene la discriminación de extranjeros en quienes son víctimas de tales agresiones. El maltrato interfiere notoriamente en su integración social y cultural, hace que inmigrantes enfaticen sus raíces culturales, favorece la nostalgia y evita que los afectados desarrollen proyectos personales en el país de acogida. Por otra parte, llama mucho la atención que las experiencias de discriminación de los hijos de inmigrantes se encuentren prácticamente limitadas al contexto escolar chileno. Esto debe hacernos reflexionar y revisar urgentemente las dinámicas escolares que se están generando en torno al fenómeno de la migración en Chile.

\section{REFERENCIAS}

Auernheimer, Georg. 1988. Der sogenannte kulturkonflikt. Orientierungsprobleme ausländischer jugendlicher. New York: Campus Verlag. .2007. Einführung in die interkulturelle pädagogik. Darmstadt: WBG.

Bechman, Torben y Sven Moerch. 2006. Transitions to work of young People with an ethnic minority or migrant background. Upf2youth -youth as actor of social change. Documento de Trabajo. 
Bendit, René. 1997. Wir wollen unsere zukunft sichern. Der zusammenhang von beruflicher ausbildung und lebensbewältigung bei jungen arbeitsmigranten in Deutschland. Aachen: Shaker Verlag.

2006. "Migration - ein steiniger Weg? Jugendliche als akteure zwischen politik und eigenen Wünschen.” DJI Bulletin 76:21-23.

Bertaux, Daniel. 2005. Los relatos de vida. Perspectiva etnosociológica. Barcelona: Ediciones Bellaterra.

Bolzman, Claudio, Rosita Fibbi y Marie Vial. 2005. "Bildungsprozesse und berufliche Integration der, Zweiten Generation'. Die Rolle der intergenerationellen Beziehungen.” Pp. 83-102 en Migration und Bildung. Über das Verhältnis von Anerkennung und Zumutung in der Einwanderungsgesellschaft, editado por F. Hamburger, T. Badawia y M. Mummrich. Wiesbaden: Verlag für Sozialwissenschaften.

Comisión Económica para América Latina y el Caribe, Centro Latinoamericano de Demografía (CEPAL/CELADE). 2006. Migración internacional. Santiago: Observatorio Demográfico América Latina y el Caribe.

Cornejo, Marcela. 2006. "El enfoque biografico: trayectorias, desarrollos teóricos y perspectivas." Psykhe 15 (1):95-106.

Cornejo, Marcela, Francisca Mendoza y Rodrigo Rojas. 2008. "La investigación con relatos de vida: pistas y opciones del diseño metodológico.” Psykhe 17 (1):29-39.

Departamento de Extranjería y Migración. 2010. Informe Anual del Departamento de Extranjería y Migración. Consultado mayo 25, 2011 (http://www.extranjeria.gov.cl/filesapp/Informe\%20Estimacion\%20Poblacion\%20Extranjeros\%202008.pdf ).

Doña, Cristián y Amanda Levinson. 2004. "Chile: moving towards a migration policy." Migration Policy Institute. Consultado septiembre 21, 2010 (http://www.migrationinformation.org/ Feature/display.cfm?ID=199).

Flick, Uwe. 2004. Introducción a la investigación cualitativa. Madrid: Ediciones Morata. Coruna: Fundacion Paideia Galiza.

Godoy, Lorena. 2007. "Fenómenos migratorios y género: identidades femeninas remodeladas." Psykhe 16 (1):41-51. 
Gutiérrez-Rodríguez, Encarnación. 1999. Intellektuelle Migrantinnen. Subjektivitäten im Zeitalter der Globalisierung. Eine postkoloniale dekonstruktive Analyse von Biographie im Spannungsverhältnis von Ethnisierung und Vergeschlechtlichung. Opladen: Leske + Budrich.

Heckmann, Friedrich. 1981. Die Bundesrepublik: Ein Einwanderungsland? Zur Soziologie der Gastarbeiterbevölkerung als Einwandererminorität. Stuttgart: Klett-Cotta.

Hein, Kerstin. 2006. Hybride Identitäten. Bastelbiografien im Spannungsverhältnis zwischen Lateinamerika und Europa. Bielefeld: transcript.

Hein, Kerstin y Ana Cárdenas. 2009. "Perspectivas de juventud en el imaginario de la política pública.” Última Década 30:95-120.

Hopf, Christel. 2000. “Qualitative Interviews - Ein Überblick.” Pp. 349-60, en Qualitative Forschung: Ein Handbuch, editado por U. Flick, E.von Kardorff e I. Steinke. Hamburg: Rowohlt.

Instituto Nacional de la Juventud (Injuv). 2006. Quinta Encuesta Nacional de Juventud. Santiago: Injuv.

International Organization for Migration (IOM/OIM). 2004. Estudio de caracterización sociodemográfica de la migración argentina en Chile. Santiago: OIM.

Lamnek, Siegfried. 1998. Qualitative Sozialforschung. Weinheim: Beltz Psychologie Verlagsunion.

Marbach, Jan. 2006. "Wie deutsch ist deutsch? Zur Bedeutung des Migrationshintergrundes." DJI Bulletin 76:8-9.

Martínez, Jorge. 2003a. Breve examen de la inmigración en Chile según los datos generales del censo de 2002. Santiago: OIM.

. 2003b. El encanto de los datos. Sociodemografía de la inmigración en Chile según el censo de 2002. Santiago: Naciones Unidas.

Mayring, Philipp. 2003. Qualitative Inhaltsanalyse. Grundlagen und Techniken. Weinheim: Beltz.

Müller, Matthias y Frank Braun. 2007. Lokales Übergangsmanagement - Handlungsbedarf und Handlungsspielräume. München: DJI. Wissenschaftliche Texte. 
Ojeda-Ebert, Gerardo. 1984. Deutsche Einwanderung und Herausbildung der chilenischen Nation (1846-1920). Beiträge zur Soziologie und Sozialkunde Lateinamerikas. Bd. 30. München: Wilhelm Fink Verlag.

Programme for International Student Assessment (PISA). 2006. Where immigrant students succeed. A comparative review of performance and engagement in PISA 2003. OECD Publishing. Portes, Alejandro y Rubén Rumbaut. 2006. Immigrant America: a portrait. Berkeley, Los Angeles, London: University California Press.

Reißig, Birgit, Nora Gaupp y Tilly Lex. 2008. Hauptschüler auf dem Weg von der Schule in die Arbeitswelt. Übergänge in Arbeit. München: DJI.

Rodríguez-Gómez, Gregorio, Javier Gil y Eduardo García. 1996. Metodología de la investigación cualitativa. Bilbao: Universidad de Deusto.

Rosenthal, Gabriele y Wolfram Fischer-Rosenthal. 2000. "Analyse narrativ-biographischer interviews." Pp. 456-68, en Qualitative orschung: Ein Handbuch, editado por U. Flick, E.von Kardorff e I. Steinke. Hamburg: Rowohlt.

Rosenthal, Gabriele. 2005. Interpretative sozialforschung. Eine einführung. München: Juventa.

Rosenthal, Gabriele, Michaela Köttig, Nicole Witte y Anne Blezinger. 2006. Biographischnarrative Gesprache mit Jugendlichen. Chancen fur das Selbst- und Fremdverstehen. Opladen: Verlag Barbara Budrich.

Salazar, Gabriel y Julio Pinto. 1999. Historia contemporánea de Chile II. Actores, identidad y movimiento. Santiago: LOM Ediciones.

Stefoni, Carolina. 2003. Inmigración peruana en Chile. Una oportunidad a la integración. Santiago: Flacso, Universitaria.

Stefoni, Carolina, Elaine Acosta, Marcia Gaymer y Francisca Casas-Cordero. 2009. Niños y niñas inmigrantes en Santiago de Chile. Entre la integración y la exclusión. Santiago: Universidad Alberto Hurtado y Organización Internacional para las Migraciones.

Straus, Florian. 1994. Netzwerkanalyse - Egozentrierte netzwerkkarten als instrument zur erhebung von sozialen beziehungen in qualitativen interviews. München: Ludwig-MaximiliansUniversität.

. 2002. Netzwerkanalysen. Gemeindepsychologische Perspektiven für Forschung und Praxis. Wiesbaden: Deutscher Universitätsverlag. 
Suanet, Irina y Fons J. R. Van de Vijver. 2009. "Perceived cultural distance and acculturation among exchange students in Russia." Journal of Community \& Applied Social Psychology 19:182-97.

Teo, Thomas. 1994. “Zur Identiät der sogenannten Mischlingen.” Pp. 145-65 en Andere Deutsche. Zur Lebenssituation von Menschen multiethnischer und multikultureller Herkunft, editado por P. Mecheril y T. Teo. Berlin: Dietz.

Walther, Andreas y Barbara Stauber. 2002. Misleading Trajectories, Integration Policies for Young Adults in Europe? Opladen: Leske + Budrich.

Ward, Colleen, Stephen Bochner y Adrian Furnham. 2001. The Psychology of Culture Shock. East Sussex, Philadelphia, New York: Routledge. 